\title{
THE EFFECTS OF VARYING OXYGEN TENSIONS UPON BONE RESORPTION IN VITRO
}

\author{
D. H. GRAY, J. M. KATZ, K. SPEAK \\ From the Department of Surgery, University of Auckland
}

\begin{abstract}
Calvaria from six-day-old infant mice were grown on a grid culture in a chemically defined medium under varying oxygen tensions. Quantitative isotope studies demonstrated a linear association between bone resorption and oxygen tension in the physiological range. This result was supported by histological, histochemical and vital staining experiments. The clinical finding of osteoporosis in areas of hyperaemia could therefore be attributed to a rise in oxygen tension causing increased bone resorption.
\end{abstract}

There have been many attempts to define the effects of varying oxygen tensions on the physiology of bone. Experiments involving the use of arteriovenous fistulae (Brookes 1972) or immobilisation (Hardt 1972) are difficult to interpret because of the associated changes in $\mathrm{pH}$ and $\mathrm{PCO}_{2}$ as well as $\mathrm{PO}_{2}$. For this reason many investigators have turned to in vitro experiments where the oxygen tension can be changed while holding other factors constant. Goldhaber (1958), Stern, Glimcher and Goldhaber (1966), Shaw and Bassett (1967) and Brighton and Schaffzin (1970) showed an increase in bone resorption under conditions of hyperoxia. On the other hand Goldhaber (1961), Melcher and Hodges (1968) and Asher and Sledge (1968) could demonstrate no clear effect on bone resorption with hyperoxia. These investigators used a variety of media, some of which contained natural tissue extracts, and different types of culture systems. The explants also varied, some containing mixtures of bone and cartilage. It was therefore decided to reinvestigate the correlation between bone resorption and oxygen tension by using a standard grid culture system, and an explant containing essentially no cartilage. A chemically defined medium was used without the addition of natural tissue extracts, as the properties of the latter may be changed by varying the oxygen tension.

\section{METHODS}

Calvaria from six-day-old infant white mice were grown on steel mesh grids in Petri dishes containing 5 millilitres of medium 199 (Commonwealth Serum Laboratories). The platforms of the grids were flush with the level of medium so that constant exposure of the explant to the liquid and gas phase was obtained. The medium was supplemented with penicillin (200000 units per litre), streptomycin (100 milligrams per litre) and vitamin C (100 milligrams per litre). The bones were grown for forty-eight hours at 37 degrees Celsius in sealed Macintosh and Fieldes jars which had been flushed with 40 litres of gas of known oxygen concentration. The gas mixtures were checked by mass spectrometry: all contained 5 per cent carbon dioxide and 5, 10,
15,20 or 25 per cent oxygen respectively, the balance being nitrogen. The medium contained 27 milliequivalents per litre of sodium bicarbonate to give a medium $\mathrm{pH}$ of 7.4 .

Two gas mixtures were studied with each litter of eight to thirteen animals. One of the two halves of the calvarium of each animal (consisting of the parietal and frontal bones) was explanted in each of the gas atmospheres under investigation in that experiment. Bones for histological examination were fixed in Zenker's fluid and stained with haematoxylin and eosin, while others were fixed in 70 per cent alcohol and stained by a standard von Kossa technique. In some experiments neutral red to a final concentration of 1 to 10000 was added to the medium at the end of the forty-eight-hour culture period, and the bones then reincubated for a further two hours in the same gas atmosphere before being examined directly at low magnification. Dye uptake by osteoclasts has been demonstrated by Barnicot (1947) using this technique.

In some experiments, based on the methods of Reynolds and Dingle (1970), two-day-old infant mice were injected subcutaneously with 10 microcuries of sterile ${ }^{45} \mathrm{CaCl}_{2}$ (Radiochemical Centre, Amersham) and the forty-eight-hour culture carried out four days later. After this the bones were decalcified in 0.5 millilitre of 90 per cent formic acid for twenty-four hours. Duplicate 0.1 millilitre aliquots of this solution and duplicate 0.5 millilitre samples of medium were then processed by standard liquid scintillation counting techniques. Appropriate calculations allowed the total isotope released from the bone into the medium, and the isotope remaining in the bone at the end of the culture, to be estimated. The sum of these two values gave the total isotope initially in the bone. To allow comparison of the absolute values obtained between animals, all the results were scaled by simple proportion to a common base of 150 nanocuries of calcium-45 in each bone initially. In every litter two or three pairs of explants were killed by triple freezing and thawing to allow estimation of the passive release of label from the bone into the medium. By subtracting this value from the total isotope in the medium, the isotope released by active bone resorption was obtained. This was expressed as a percentage of the isotope contained originally in the bone (Reynolds et al. 1972).

\section{RESULTS}

Histology. Bones grown in 20 per cent oxygen demonstrated well-preserved cell morphology. These appearances were maintained in bones grown in 10 per cent oxygen. There appeared to be some reduction in cell
D. H. Gray, Ch.M., M.Med.Sci., F.R.A.C.S., Professor of Orthopaedic Surgery

J. M. Katz, M.Sc., Technician

K. Speak, N.Z.C.S., Technician
The Department of Surgery, School of Medicine, University \} of Auckland, Private Bag, Auckland, New Zealand. 
numbers in 5 per cent oxygen, but cell morphology was still satisfactory.

Von Kossa staining. This showed some inhibition of resting resorption in 10 per cent oxygen as opposed to 20 per cent oxygen, with the difference between 10 per cent pooled (Table II) and shown in graphical form in Figure 1 , except that series 6 has been excluded as the gas tensions studied were not related to adjacent points on the curve. It will be observed that eight bones grown in 5 per cent carbon dioxide and 95 per cent nitrogen

Table I. Mean values for bone resorption

\begin{tabular}{|c|c|c|c|c|c|c|c|c|c|c|}
\hline \multirow[b]{2}{*}{ Series } & \multicolumn{3}{|c|}{ Oxygen tension* } & \multicolumn{2}{|c|}{ Total resorption } & \multicolumn{2}{|c|}{ Passive resorption } & \multicolumn{2}{|c|}{ Active resorption } & \multirow[b]{2}{*}{$t$ test } \\
\hline & $\%$ & $\mathrm{mmHg}$ & $\mathrm{kPa}$ & $n C i$ & (n) & $\begin{array}{c}\text { Observed } \\
n C i\end{array}$ & $n C i^{\text {Mean }}(n)$ & $n C i$ & $\%$ & \\
\hline 1 & 0 & 0 & 0 & 31.47 & (8) & 31.27 & $31.27 \quad(8)$ & 0.20 & 0.13 & \\
\hline 2 & $\begin{array}{r}10 \\
5\end{array}$ & $\begin{array}{l}71 \\
36\end{array}$ & $\begin{array}{l}9 \\
5\end{array}$ & $\begin{array}{l}34.76 \\
28.66\end{array}$ & $\begin{array}{l}(24) \\
(24)\end{array}$ & $\begin{array}{l}23.47 \\
22.55\end{array}$ & \begin{tabular}{|ll} 
& $(9)$ \\
23.01 & $(9)$
\end{tabular} & $\begin{array}{r}11.75 \\
5.65\end{array}$ & $\begin{array}{l}7.83 \\
3.77\end{array}$ & $P<.001$ \\
\hline 3 & $\begin{array}{l}15 \\
10\end{array}$ & $\begin{array}{r}107 \\
71\end{array}$ & $\begin{array}{r}14 \\
9\end{array}$ & $\begin{array}{l}43.52 \\
34.93\end{array}$ & $\begin{array}{l}(19) \\
(19)\end{array}$ & $\begin{array}{l}22.73 \\
19.59\end{array}$ & $\begin{array}{ll}21.16 & (9) \\
& (9)\end{array}$ & $\begin{array}{l}22.36 \\
13.77\end{array}$ & $\begin{array}{r}14.91 \\
9.18\end{array}$ & $P<.001$ \\
\hline 4 & $\begin{array}{l}20 \\
15\end{array}$ & $\begin{array}{l}143 \\
107\end{array}$ & $\begin{array}{l}19 \\
14\end{array}$ & $\begin{array}{l}49.04 \\
47.57\end{array}$ & $\begin{array}{l}(23) \\
(23)\end{array}$ & $\begin{array}{l}26.22 \\
28.43\end{array}$ & $27.32(11)$ & $\begin{array}{l}21.72 \\
20.25\end{array}$ & $\begin{array}{l}14.48 \\
13.50\end{array}$ & $P<.20$ \\
\hline 5 & $\begin{array}{l}25 \\
20\end{array}$ & $\begin{array}{l}178 \\
143\end{array}$ & $\begin{array}{l}24 \\
19\end{array}$ & $\begin{array}{l}51.43 \\
49.68\end{array}$ & $\begin{array}{l}(21) \\
(21)\end{array}$ & $\begin{array}{l}24.67 \\
25.40\end{array}$ & $25.04(12)$ & $\begin{array}{l}26.39 \\
24.64\end{array}$ & $\begin{array}{l}17.59 \\
16.43\end{array}$ & $P<.25$ \\
\hline 6 & $\begin{array}{l}25 \\
15\end{array}$ & $\begin{array}{l}178 \\
107\end{array}$ & $\begin{array}{l}24 \\
14\end{array}$ & $\begin{array}{l}58.12 \\
50.94\end{array}$ & $\begin{array}{l}(18) \\
(18)\end{array}$ & $\begin{array}{l}30.13 \\
28.22\end{array}$ & \begin{tabular}{|ll} 
& $(6)$ \\
29.17 & $(6)$
\end{tabular} & $\begin{array}{l}28.95 \\
21.77\end{array}$ & $\begin{array}{l}19.30 \\
14.51\end{array}$ & $P<.001$ \\
\hline
\end{tabular}

$\mathrm{nCi}$ expressed as $\mathrm{nCi} / 150 \mathrm{nCi}$ in bone initially.

*all gas mixtures contained 5 per cent carbon dioxide, the stated amount of oxygen and the balance as nitrogen.

and 5 per cent oxygen being less evident. There was qualitative evidence for increased bone resorption with increased oxygen tension.

Neutral red studies. The osteoclasts were seen to take up the dye readily after growth in 20 per cent oxygen, but there was a slightly smaller uptake in 10 per cent oxygen and less in 5 per cent oxygen, although the differences were not marked.

Isotope studies. In Table I the mean values for bone resorption are shown for each series. The dead bone values were not significantly different when any two atmospheres were compared. Minor, although insignificant, differences in these values could have a marked effect on the calculated cell-mediated activity when the values are of borderline significance. Therefore, the dead bone values were averaged within each series. Failure to do this would have resulted in a finding of false-positive differences in bone resorption between 15 and 20 per cent oxygen, and 20 and 25 per cent oxygen. The differences in these series were below the resolution of the method and were not significant. There were significant increases in bone resorption between 5 and 10 per cent oxygen, between 10 and 15 per cent oxygen, and between 15 and 25 per cent oxygen. All the results obtained for each gas atmosphere have been behaved as dead bone. There was a linear increase in bone resorption in atmospheres up to 15 per cent oxygen (14 kilopascals); at higher concentrations bone resorption continued to increase, but at a slower rate. The significant differences in resorption between 15 and 25 per cent oxygen accounted for this continued upward slope of the curve at these tensions even though the differences individually between 15 and 20 per cent, and between 20 and 25 per cent oxygen were not significant.

Table II. Active resorption for each gas atmosphere

\begin{tabular}{|r|c|c|cr|}
\hline \multicolumn{3}{|c|}{ Oxygen tension* } & \multicolumn{2}{c|}{ Active resorption } \\
\hline$\%$ & $m m H g$ & $k P a$ & $\% \pm s . d$. & $(n)$ \\
\hline 0 & 0 & 0 & 0.13 & $(8)$ \\
5 & 36 & 5 & $3.49 \pm 0.84$ & $(24)$ \\
10 & 71 & 9 & $8.24 \pm 2.58$ & $(43)$ \\
15 & 107 & 14 & $14.20 \pm 2.15$ & $(42)$ \\
20 & 143 & 19 & $15.55 \pm 2.32$ & $(44)$ \\
25 & 178 & 24 & $17.72 \pm 3.17$ & $(21)$ \\
\hline
\end{tabular}

*all gas mixtures contained 5 per cent carbon dioxide, the stated amount of oxygen and balance as nitrogen. 


\section{DISCUSSION}

The histological and vital staining studies indicated that the explants showed good viability over the range of oxygen tensions studied. This was in contrast to the findings of Gray and Hamblen (1976) who saw marked toxic changes histologically, and by vital staining, when calvaria were exposed to oxygen at one and two atmospheres. The increased bone resorption suggested qualitatively by the von Kossa studies, was confirmed in the quantitative isotope measurements which showed a linear association between bone resorption and oxygen tension up to 14 kilopascals. This latter corresponded to the oxygen tension seen in the medullary canal of the long bones of experimental animals (Brighton and Heppenstall 1971). This is the first report in which bone resorption has been quantified over the physiological range of oxygen tension.

Bone cells will never be exposed to a higher oxygen tension than arterial $\mathrm{PO}_{2}$ in vivo (14 kilopascals) but will be exposed to some undetermined lower level, at which changes in oxygen tension would lead to proportional changes in bone resorption. At oxygen tensions slightly higher than those normally encountered in vivo, the bone resorption rate continues to increase, but at a slower rate.

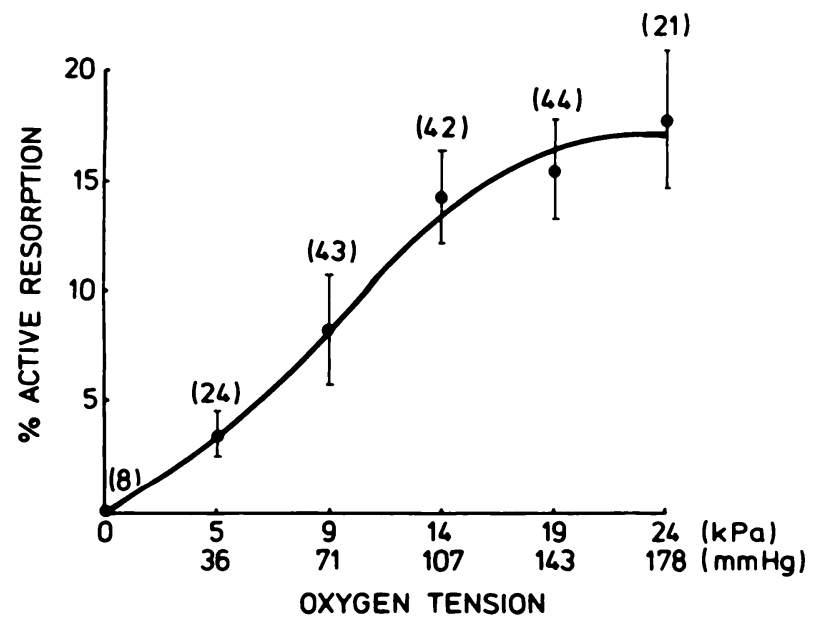

Fig. 1

This graph relating oxygen tension and active bone resorption is derived from Table II. The curve is virtually linear in the physiological range below 14 kilopascals. The vertical bars represent one standard deviation on each side of the mean.

The previous studies of Gray and Hamblen (1976) using the same culture system have shown a marked inhibition of bone resorption at oxygen tensions of one and two atmospheres, due to toxic changes at these levels. The graph of bone resorption against oxygen tension therefore follows a skewed bell-shaped curve with the peak in the range between 24 and 100 kilopascals (Fig. 2). An explanation for the conflicting results obtained by previous investigators is now possible. The point on the curve at which any experimental design starts will be governed by the physical nature of the culture system, the content of the medium and the composition of the explants. If the starting point in any one system is to the left of the mode, an initial increase in oxygen tension would result in an

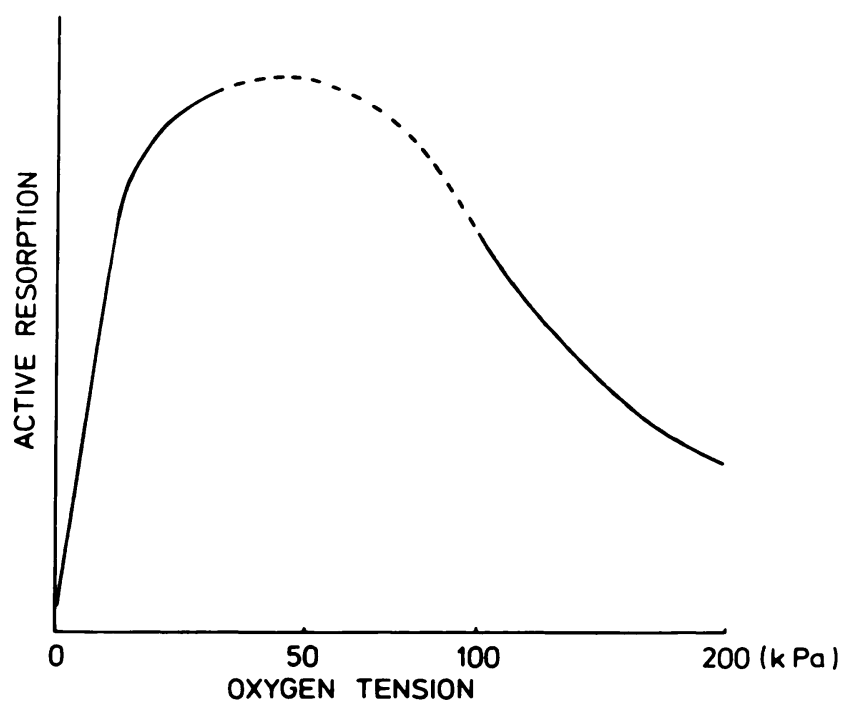

Fig. 2

This curve is derived from the results of the present investigation and those of Gray and Hamblen (1976) as described in the text. The numerical value of the mode has not been determined.

increase in bone resorption, but on the other hand if the starting point is at or near the mode an increase in oxygen tension will result in a decrease in bone resorption.

Previous studies (Borle, Nichols and Nichols 1960) have shown that the metabolism of bone cells is largely anaerobic, following glycolytic pathways. However, total anoxia results in cell death as has been shown here. It is uncertain to what extent the increased bone resorption found with increasing oxygen tension is due to activation of non-specific oxygen-dependent enzyme systems, rather than to a specific effect of oxygen upon bone resorptive mechanisms. Quantitative information, using this model to demonstrate the effects of oxygen upon bone formation, would help to resolve this dilemma and investigations are under way in this laboratory. These experiments will also determine whether increasing oxygen tension differentially affects formation and resorption or merely increases bone turnover.

Although care must be taken in projecting the findings in vitro to the possible clinical state in vivo, our results provide a satisfactory explanation for some commonly observed clinical phenomena. Bone resorption is often seen radiographically adjacent to foci of inflammation or infection and around some fractures. If these disorders give rise to a state of localised hyperaemia with an increased oxygen tension, then on 
the basis of the present findings one would expect increased bone resorption to occur. The marked bone resorption accompanying the hyperaemia of Sudeck's atrophy could be explained in a similar way.

The osteoporosis associated with disuse and immobilisation has also been attributed to changes in blood gases (Hardt 1972). In that investigation, using rabbits immobilised by calcaneal tenotomy, a period of bone hyperoxia was found for up to ten days after a period of relative hypoxia. During the initial phase there was an increase in bone calcium and phosphorus and a reduction of these factors during the hypoxic phase. However, bone resorption was not measured directly and there were accompanying changes in $\mathrm{pH}$ and $\mathrm{PCO}_{2}$. Although changes in oxygen tension may be important in this phenomenon, changes in mechanical stress factors could form a more reasonable basis for these effects.

We are most grateful to the Medical Research Council of New Zealand and the Sir William Stevenson Orthopaedic Trust for generous financial support. Mr A. B. Ferguson and his staff have helped greatly with the supply of animals and Mrs Patricia James has provided excellent secretarial support. We are also indebted to Professor J. D. Sinclair and his staff in the Department of Physiology for permission to use the gas-mixing equipment.

\section{REFERENCES}

Asher, M. A., and Sledge, C. B. (1968) Hyperoxia and in-vitro bone resorption. Clinical Orthopaedics and Related Research, 61, 48-51.

Bamicot, N. A. (1947) The supravital staining of osteoclasts with neutral-red; their distribution on the parietal bone of normal growing mice, and a comparison with the mutants grey-lethal and hydrocephalus-3. Proceedings of the Royal Society of London, Series B, 134, 467-485.

Borle, A. B., Nichols, N., and Nichols, G. Jun. (1960) Metabolic studies of bone in vitro. I. Normal bone. Journal of Biological Chemistry, 235, $1206-1210$.

Brighton, C. T., and Heppenstall, R. B. (1971) Oxygen tension in zones of the epiphyseal plate, the metaphysis and diaphysis. Journal of Bone and Joint Surgery, 53-A, 719-728.

Brighton, C. T., and Schafizin, E. A. (1970) Comparison of the effects of excess vitamin A and high oxygen tension on in vitro epiphyseal plate growth. I. Morphologic growth. Calcified Tissue Research, 6, 151-161.

Brookes, M. (1972) Blood supply of developing bone and its possible bearing on malformation of the limbs and face in congenital haemangiomatous disorders. Proceedings of the Royal Society of Medicine, 65, 597-599.

Goldhaber, P. (1958) The effect of hyperoxia on bone resorption in tissue culture. Archives of Pathology, 66, 635-641.

Goldhaber, P. (1961) Oxygen-dependent bone resorption in tissue culture. In The Parathyroids, pp. 243-254. Edited by R. O. Greep and T. V. Talmage. Springfield, Illinois: Charles C. Thomas.

Gray, D. H., and Hamblen, D. L. (1976) The effects of hyperoxia upon bone in organ culture. Clinical Orthopaedics and Related Research, 119, 225-230.

Handt, A. B. (1972) Early metabolic responses of bone to immobilization. Journal of Bone and Joint Surgery, 54-A, 119-124.

Mekcher, A. H., and Hodges, G. M. (1968) In vitro culture of an organ containing mixed epithelial and connective tissues on a chemically defined medium. Nature (London), 219, 301-302.

Reynolds, J. J., and Dingle, J. T. (1970) A sensitive in vitro method for studying the induction and inhibition of bone resorption. Calcified Tissue Research, 4, 339-349.

Reynolds, J. J., Minkin, C., Morgan, D. B., Spycher, D., and Fleisch, H. (1972) The effects of two diphosphonates on the resorption of mouse calvaria in vitro. Calcified Tissue Research, 10, 302-313.

Shaw, J. L., and Bassett, C. A. L. (1967) The effects of varying oxygen concentrations on osteogenesis and embryonic cartilage in vitro. Journal of Bone and Joint Surgery, 49-A, 73-80.

Stern, B., Glimcher, M. J., and Goldhaber, P. (1966) The effect of various oxygen tensions on the synthesis and degradation of bone collagen in tissue culture. Proceedings of the Society for Experimental Biology and Medicine, 121, 869-872. 